\title{
Understandings of Dementia in Pakistan: A Project Proposal
}

Dr Nicolas Farina ${ }^{1}$, Dr Rosalind Willis², Dr Sara Balouch ${ }^{1}$, Dr Qurat Khan ${ }^{3}$, Dr Hussain Jafri ${ }^{4}$, Dr Irfan Ahmad ${ }^{5}$, and Prof Asghar Zaidi ${ }^{6}$

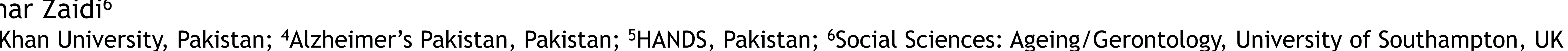
INTRODUCTION

- Dementia has become a global health priority, although progress in low and middle income countries is lacking where the speed of population ageing is faster.

There is as yet no cure for dementia, but there is evidence that formal support services can help to improve the quality of life of persons living with dementia and that of their family caregivers (Downs and Bowers, 2010; Farina et al., 2017).

It is therefore important that a diagnosis is made as early as possible, but contact with services can be delayed if individuals or family members do not recognise the symptoms of dementia.

A barrier to seeking help is the stigma associated with dementia (ADI 2012), due to underlying negative and inaccurate beliefs about it particularly that it is the individual's own fault or due to family neglect (La Fontaine et al., 2007).

In Pakistan there is limited awareness among the general public about dementia and it is often believed to occur as a part of normal ageing process, secondary to traumatic events or stressors, or physical weakness (Qadir et al., 2013).

It is essential that we identify peoples' understanding, beliefs and attitudes about dementia so as to advocate for the most effective policies and programmes in raising awareness and providing support services to people with dementia and their caregivers.

\section{PAKISTAN AT A GLANCE}

- In 2015, 11.6 million Pakistanis are over 60 years of age and this figure will rise to 43.3 million by 2050 (Zaidi, 2016).

It ranks low in the Global Age Watch Index: at 92 out of 94 countries (Zaidi, 2013).

It ranks particularly low with respect to health of older persons, with a relatively low life expectancy and even lower healthy life expectancy within the region (Zaidi, 2016)

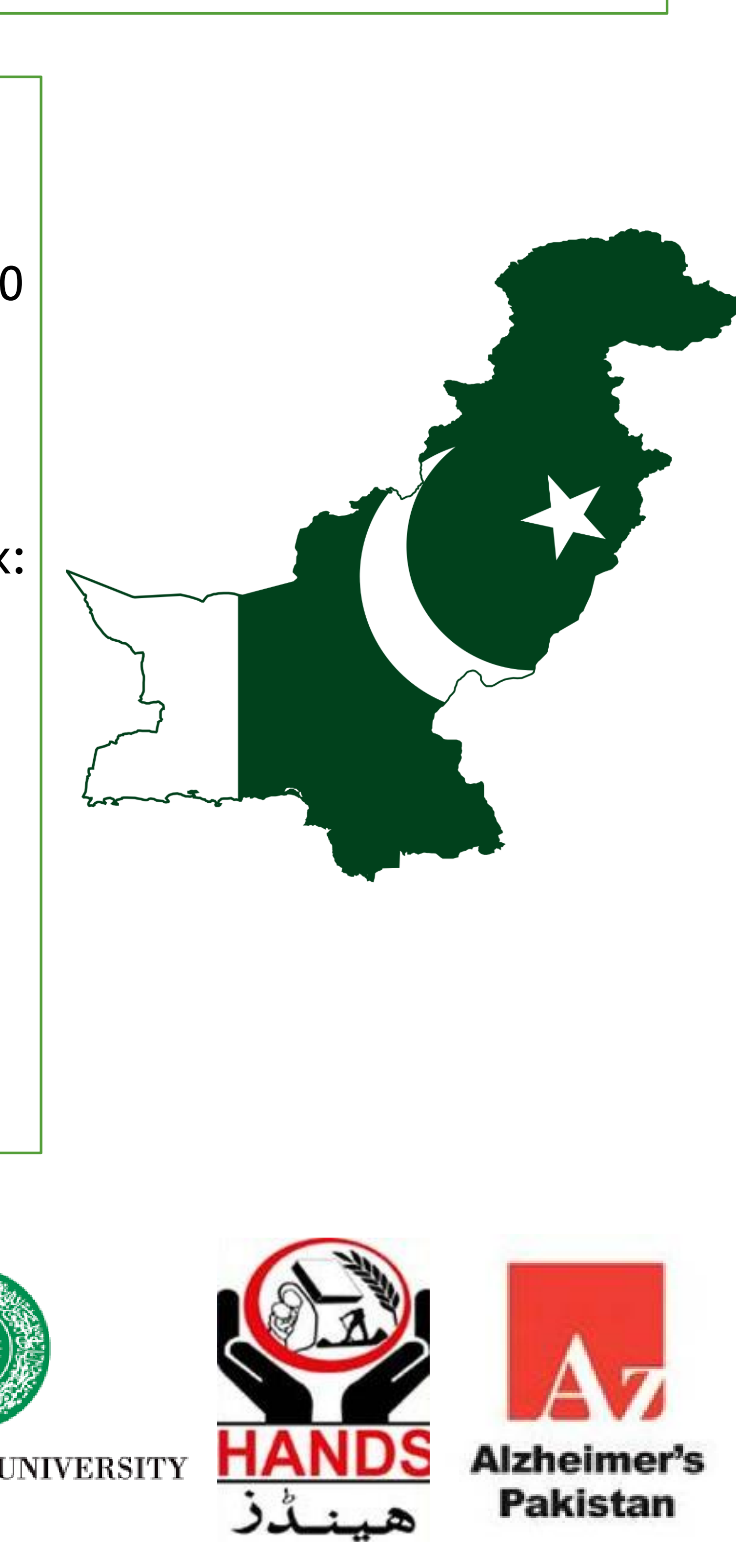

\section{Southammpton}

\section{AIMS}

To understand the cultural beliefs, attitudes, views and understanding of dementia in people living with dementia and their families in Pakistan, and in those who have no

experience of dementia.

\section{METHODS: FOCUS GROUP DISCUSSIONS (FGDs)}

- A series of FGDs will be held with members of the general public in Pakistan $(n=40)$. There will be a total of 4 single-events in Lahore and Karachi. Groups will be split by gender.

- Two vignettes will be presented to participants, describing a person with dementia at different stages of the disease (see example below). The vignettes are culturally adapted from a study that explored awareness and understanding of dementia in minority ethnic groups within the UK (La Fontaine et al., 2007).

The vignettes are independently reviewed by dementia experts (UK) and then by senio Pakistani academics and clinicians. Finally, the translated vignettes will be reviewed by a PPI group in Pakistan.

The discussion has been opened up to the group's understanding of what is happening to the people in the vignette, and probe issues concerning the cause of the condition and how to best support and treat them.

- Vignettes are useful tools for FGDs on sensitive topics, because they shift the focus away from the individual and onto the general, making it easier for group members to offer opinions.

\section{METHODS: INDIVIDUAL INTERVIEWS}

- People with mild dementia $(n=20)$ will take part in a one-off individual semi-structured interview, where they will be asked open-ended questions about their experience with their illness, their understanding of their illness, and how other people have responded to it.

Family caregivers ( $\mathrm{n}=20)$ will also take part in a one-off individual semi-structured interview, where they will be asked open-ended questions about their family member's illness, their understanding of the illness, and how they and other family members have responded to it.

\section{METHODS: KEY INFORMANT INTERVIEWS}

The Key Informant Interviews will be a one-off individual semi-structured interview with key stakeholders in Pakistan, for example practitioners and policymakers where they will be asked open-ended questions about the public policy programmes and services

currently in place in Pakistan to address the issues of people with dementia.

\section{EXAMPLE VIGNETTE}

MRS S, 72 YEARS OLD
"Mrs Shabnam used to be very proud of her appearance and was actively involved with
the family. She looked after her grandchildren happily and willingly, and never missed the family. She looked after her grandchildren happily and willingly, and never missed an opportunity of visiting other members of her extended family.

However, things have changed since she was diagnosed with diabetes 6 months ago. A couple of months later, her family began noticing that she was becoming more less pride in her appearance. She has also now lost interest in providing care for her grandchildren and does not wish to make efforts to visit family and friends. After her daughter-in-law persuaded her to attend her older grandson's wedding, it became apparent that she was having some difficulty in remembering the names of her and began discussing the last family gathering, Mrs Shabnam's 70th birthday celebration. But she seemed confused and had difficulty remembering the gathering properly and who had attended. She found it much easier to join in a conversation about a wedding in Multan 40 years earlier. To the embarrassment of the Mrs Shabna she also found herself getting lost coming back from the bathroom at the venue.

Since the wedding, the family has been talking about the changes they are observing
in her. Members of Mrs Shabnam's family are telling her daughter-in-law to take her to a local doctor; others are just saying there is nothing that can be done, it is just a part of the process of getting older."

(2)

\section{DISCUSSION}

Understanding people's attitudes of dementia in Pakistan is the first stage in reducing stigma and improve health and social care toward the condition in the country. Whilst this research is limited to Pakistan, understanding different cultural views of dementia globally is vital.

It is envisaged that there will be stigma toward dementia both from families living with the condition and also the general public in Pakistan. It is likely that better dementia education to people in Pakistan is needed to change these attitudes.
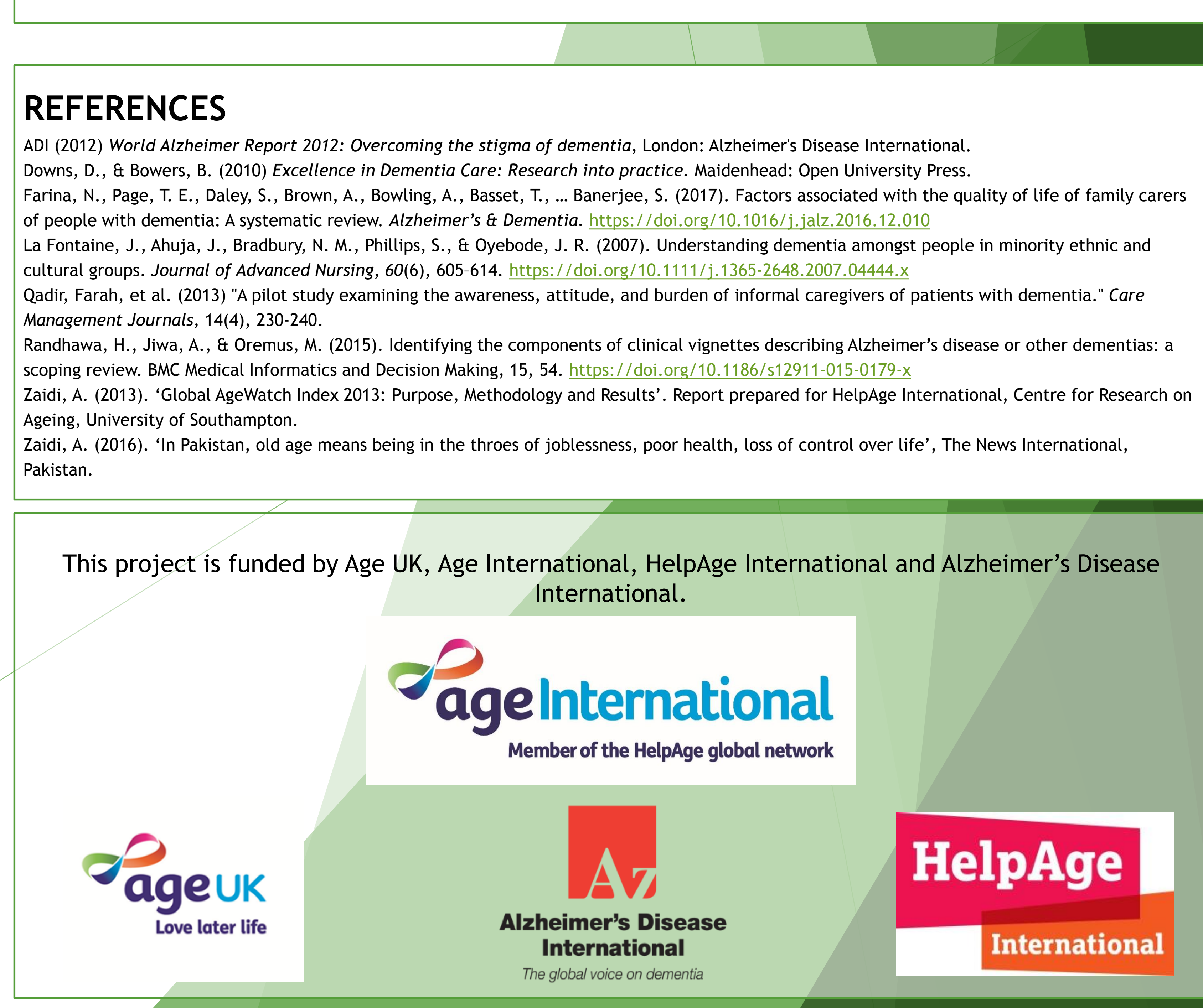